\title{
Glucose transporter 1 suppresses melanocortin 4 receptor activity
}

Anne Müller ${ }^{1}$, Lars Niederstadt², Sabine Jyrch ${ }^{1}$, Wenke Jonas ${ }^{3}$, Franziska Meyer ${ }^{4}$, Carsten Grötzinger ${ }^{2}$, Annette Schürmann $^{3}$, Gunnar Kleinau', Annette Grüters ${ }^{1}$, Heiko Krude ${ }^{1}$, and Heike Biebermann'1

${ }^{1}$ Institut für Experimentelle Pädiatrische Endokrinologie, Charité-Universitätsmedizin, Berlin, Germany; ${ }^{2}$ Department of Hepatology and Gastroenterology and Molecular Cancer Research Center (MKFZ), Tumor Targeting Lab, Charité-Universitätsmedizin Berlin, Germany; ${ }^{3}$ Department of Experimental Diabetology, German Institute of Human Nutrition (DIfE), Potsdam-Rehbruecke and German Center of Diabetes Research, Neuherberg, Germany; ${ }^{4}$ Institut für Experimentelle Endokrinologie, Charité-Universitätsmedizin Berlin, Germany

\section{Introduction and Aim of study}

Overweight, obesity and associated diseases are global issues expanding in an epidemic way. Body weight is controlled by hypothalamic $G$ protein coupled receptors (GPCRs). Especially the melanocortin 4 receptor (MC4R), expressed in the paraventricular nucleus (PVN) plays a crucial role in feeding behavior. MC4R knockout or loss-of-function variants result in hyperphagia and early-onset obesity [1]. One characteristic of GPCRs is the capability to form di- or oligomeric structures. It was demonstrated that MC4R interactions can have high impact on its signaling [2]. However, so far just a few interaction partners are known. Therefore we applied a screening based on protein complementation to detect further MCR interactions. The glucose transporter 1 was one potential MCR interactor. GLUT1 could represent a high sensitive glucose sensor at neurons that mediates the nutrition state between neurons, operating after the first important glucose maintenance by the GLUT3. A GLUT1 interaction with the most powerful food intake controlling MC4R and moreover an influence of blood glucose level on MC4R signaling (feeding behavior) would present a new neuronal circuitry. Aim of the study was to confirm and to characterize in a first step a hypothalamic MC4R/GLUT1 interplay.

\section{Result I: GLUT1 expression on PVN neurons}
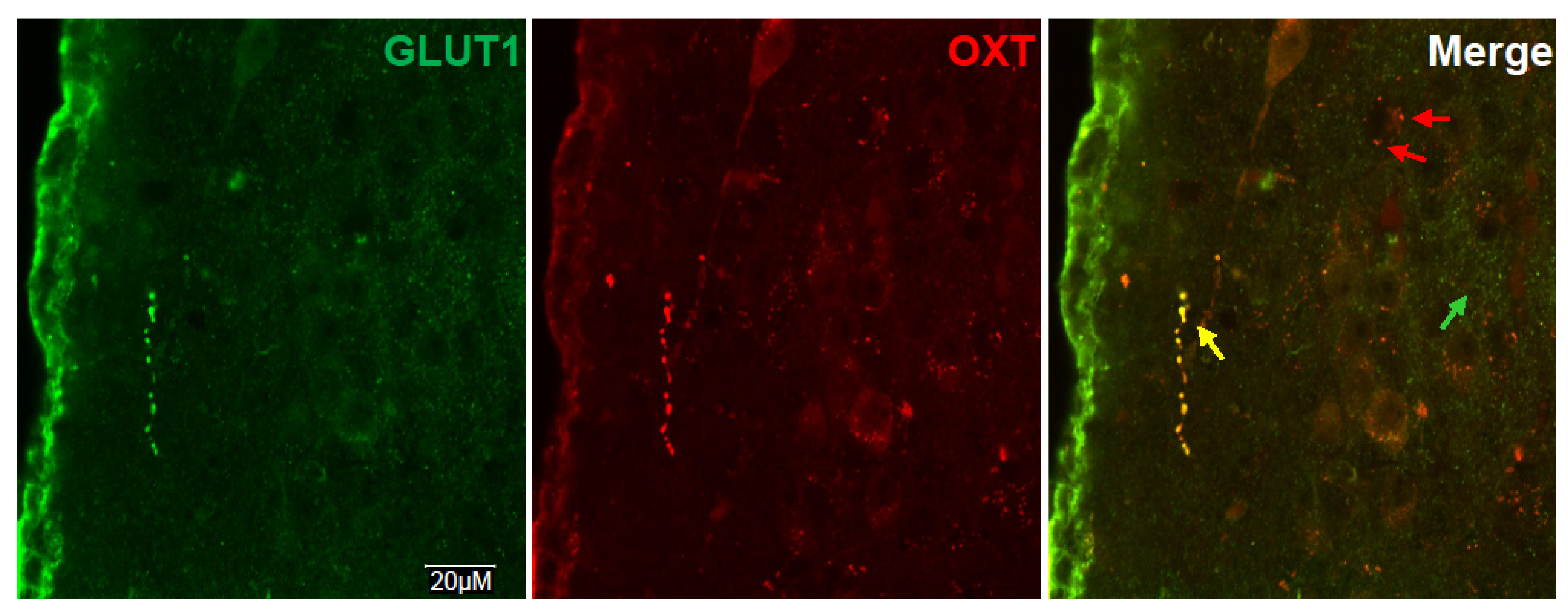

Fig.1: GLUT1 expression pattern in the PVN.

GLUT1 and oxytocin (OXT, as neuronal PVN marker) detected by immunhistochemistry. Structure (1) Merge: green arrow, exclusive GLUT1 expression; red arrows, exclusive OXT expression; yellow arrow, GLUT1/OXT co-expression.

Result III: GLUT1 interacts with the MC4R
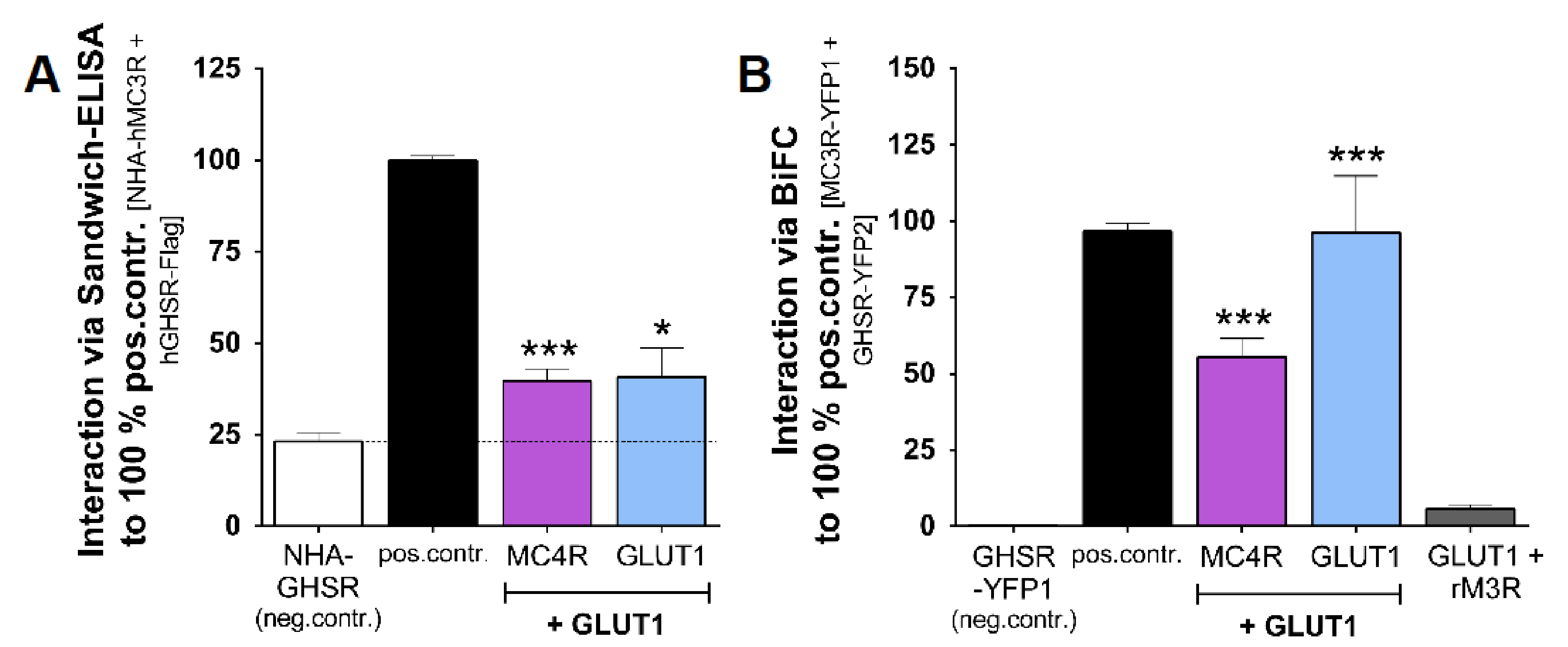

Fig.3: MC4R/GLUT1 interaction. Receptor dimerization was measured using Sandwich ELISA (A) and YFP-BiFC (B). The MC3R/GHSR hetero- [3] and the GLUT1 homodimer [4] served as positive controls, transfection of a single construct as negative control. Data from three independent experiments were calculated to $100 \%$ of $p 0$ contive control and represt.

$\rightarrow$ GLUT1/MC4R interaction is shown in two different assay systems

$\rightarrow$ GLUT1/MC4R interaction in quantity comparable to GLUT1 homodimer (Fig.3A)

Result V: Hypothalamic GLUT1 expression is down-regulated after 3 days of high-fat diet (HFD)

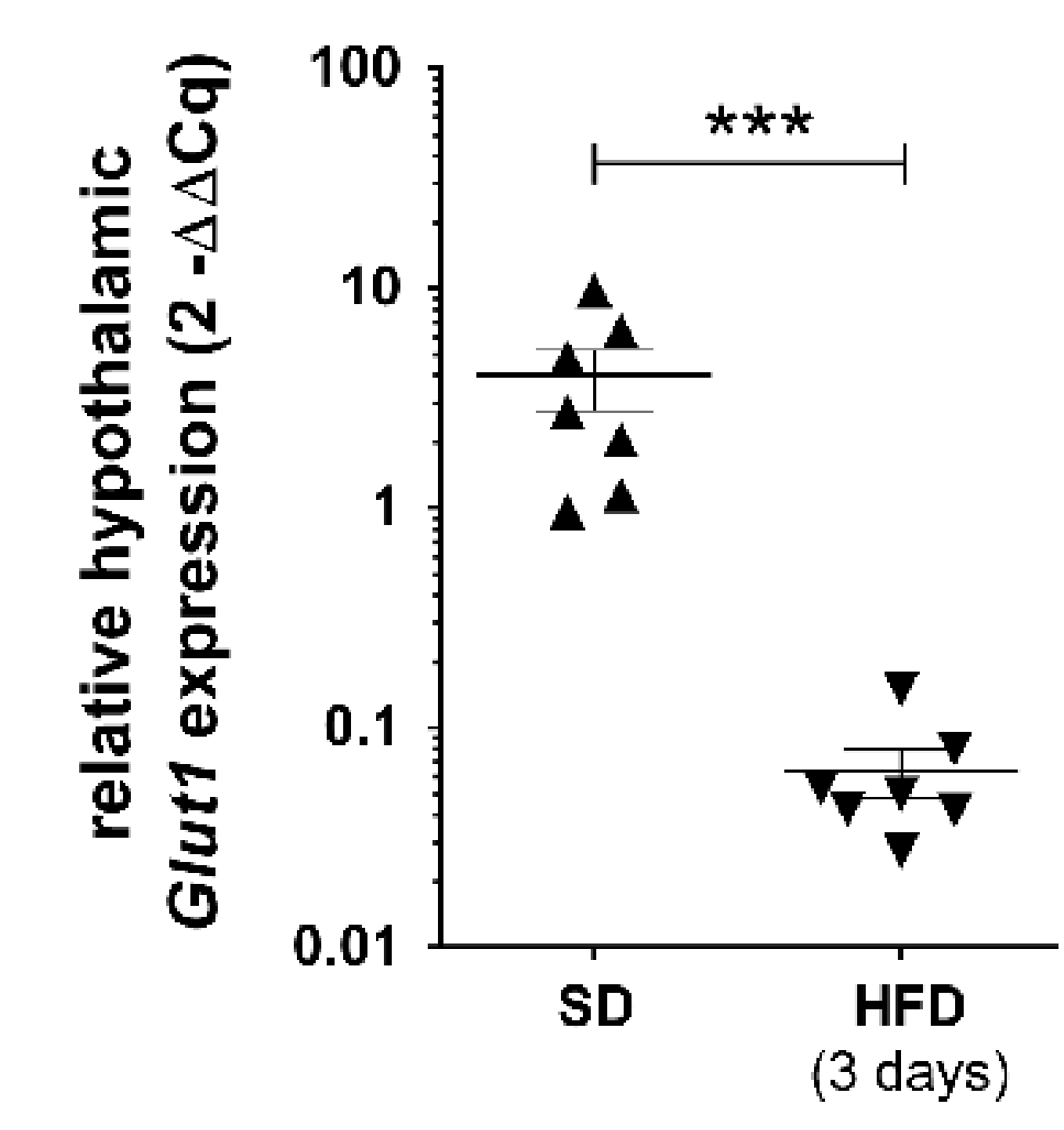

Fig.5: Hypothalamic Glut1 expression after 3 days of HFD.

Quantitative real-time PCR for 7 animals fed with standard diet, compared to 7 animals fed for three days with high-fat diet. QPCR performed in duplicates for each animal. Reference gene: $18 \mathrm{~S}$ ribosomal RNA (Rn18S) due to its minimal variation across experimental groups. Data were analyzed using the comparative Cq method and represent mean \pm SEM. ${ }^{* * *} \mathrm{p} \leq 0.001$

$\rightarrow$ Glut1 expression in HFD mice is extremely declined by $98 \%$ in comparison to SD fed animals

[1] D. Huszar et al., Cell (1997), [2] L.F. Chan et al., Proc Natl Acad Sci U S A (2009), [3] A. Rediger et al., Obes Facts (2009), [4] D.N. Hebert et al., Biochemistry (1991), [5] A. Elsner et al., Peptides (2006), [6] A.S. Garfield et al
Result II: GLUT1/MC4R co-expression on neuronal cell lines

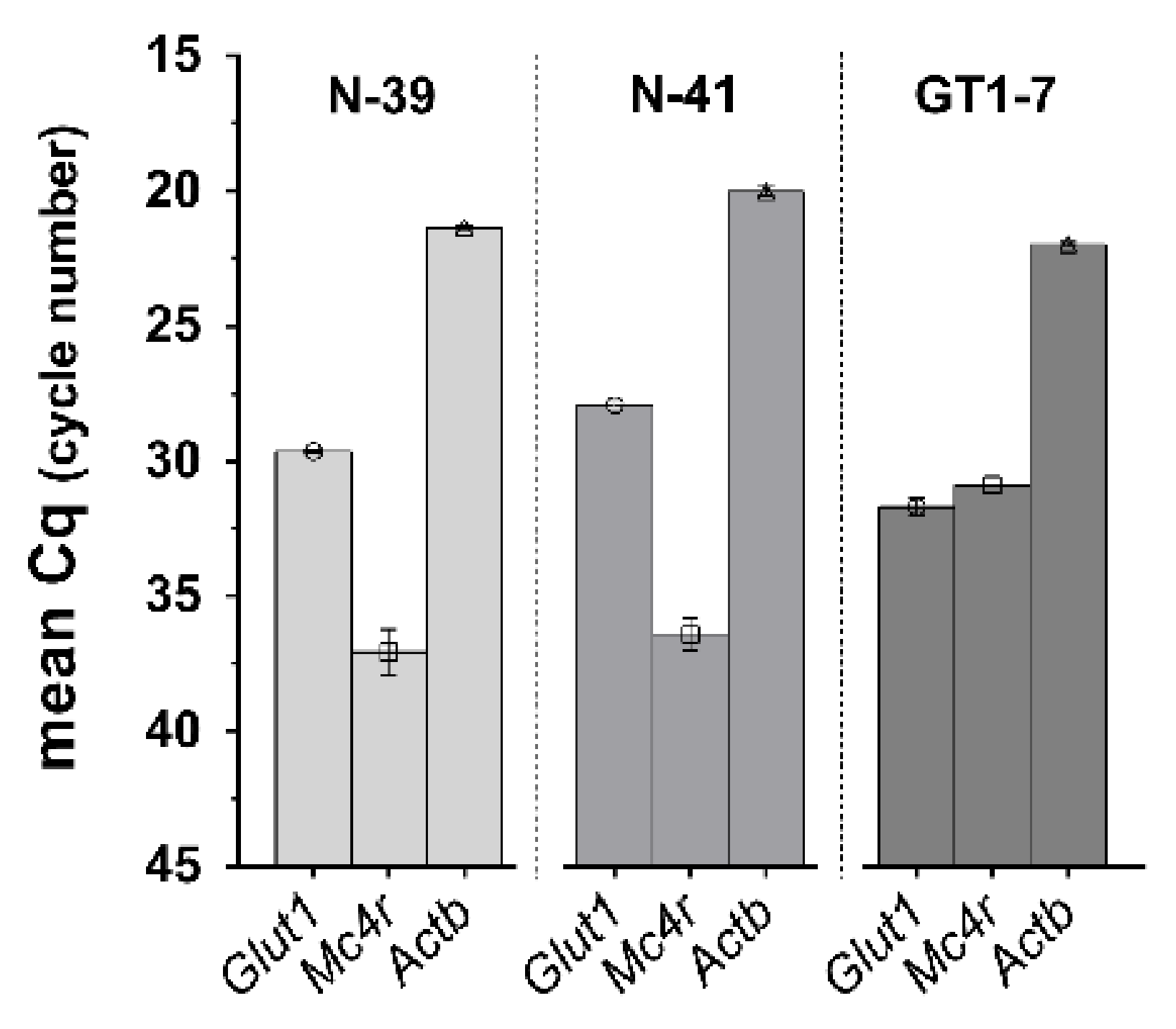

Fig.2: GLUT1 and MC4R expression in different

hypothalamic murine neuronal cell lines. Quantitative real-time PCR. Reference gene: beta-actin (Actb). Data represent mean Cq values.

$\rightarrow$ GLUT1 is strongly expressed on cells lining the third ventricle (Fig.1)

$\rightarrow$ GLUT1 is expressed on OXT expressing PVN neurons (Fig.1, Merge: yellow signals)

$\rightarrow$ GLUT1 is also expressed on PVN cells that don't express OXT (Fig.1, Merge: green signals)

$\rightarrow$ GLUT1 and MC4R are co-expressed on neurons (Fig.2)

Result IV: GLUT1 inhibits MC4R signaling and expression
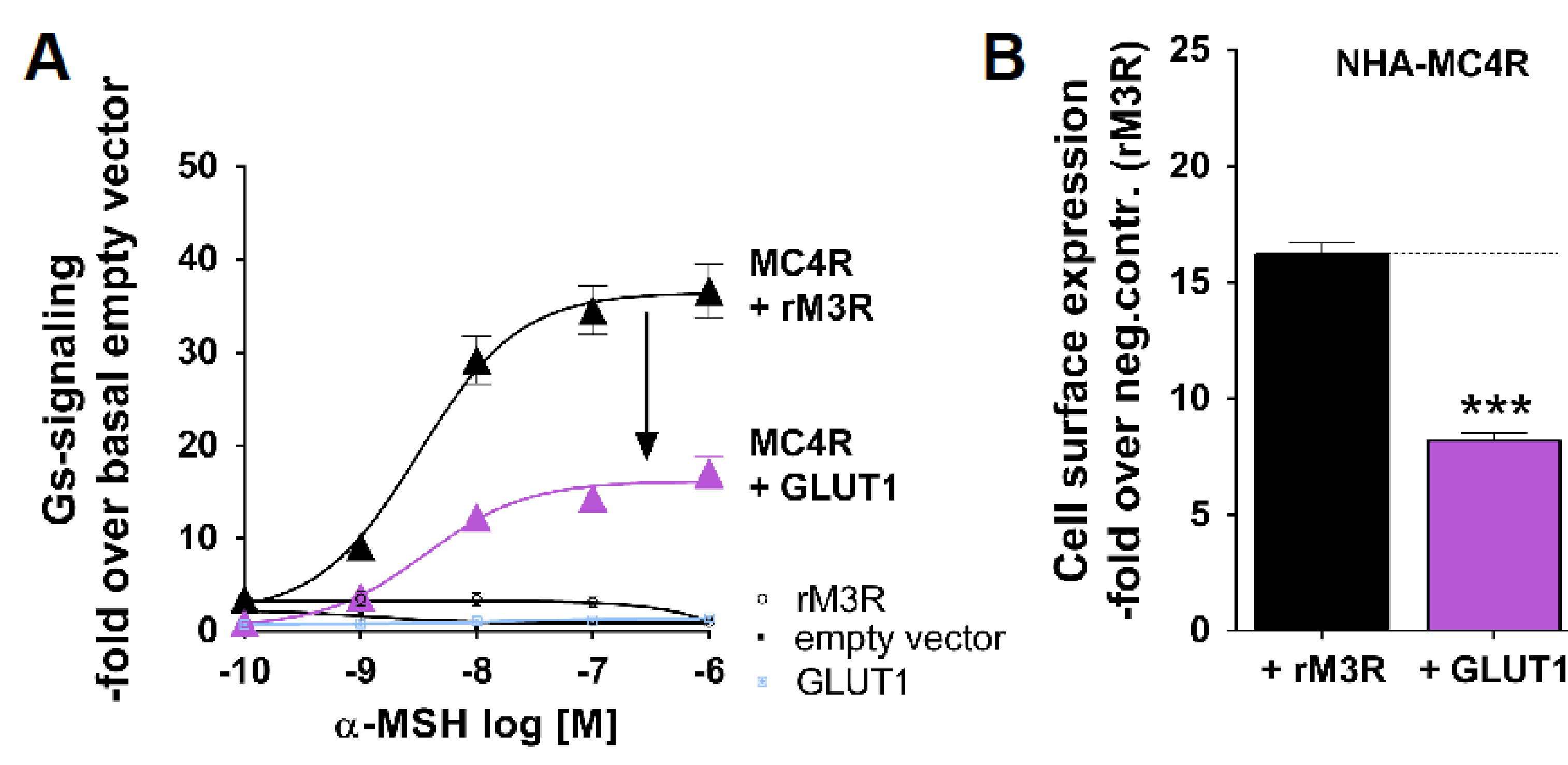

Fig.4: Impact of GLUT1 on MC4R function. Gs-activity (A, CAMP-accumulation) was measured using AlphaLISA-technology. Cell surface expression (B) was determined in an ELISA detecting the HA-tag. MC4R coexpressed with the non-interactive rM3R [5] (black) in comparison to the MC4R/GLUT1 interplay (violet). Data from three independent experiments, calculated to the stated control. \pm and + SEM. ${ }^{\star \star *} p \leq 0.001$ (arrow).

$\rightarrow$ GLUT1 reduces MC4R signaling to $42 \%$ without an effect on EC50 (Fig.4A) $\rightarrow$ Cell surface expression of MC4R is reduced to $50 \%$ by GLUT1 (Fig.4B)

\section{Discussion}

In the present study we could show GLUT1 expression on oxytocin expressing and non-oxytocin (supposed to be the MC4R expressing ones [6]) PVN neurons. In addition we demonstrated GLUT1/MC4R co-expression in three neuronal cell lines (Fig.2) facilitating a possible physiological MC4R/GLUT1 correlation. Further experiments confirmed a direct MC4R/GLUT1 interaction (Fig.3) and a GLUT1 mediated inhibition of MC4R signaling by reduction of MC4R cell surface expression (Fig.4). Analyzing this receptor/transporter relationship in the background of high-fat diet we found strong down-regulation of GLUT1 expression after three days of HFD (Fig.5) representing a very physiological reaction at this point of research. HFD should mediate satiation. By decreasing GLUT1 expression in response to HFD the MC4R obtains higher cell surface expression and activity reducing food intake. By these data a new level of MC4R function was determined. Further studies could reveal if the GLUT1 expression is increasing after a longer period of HFD contributing to the development of overweight and obesity. 